\title{
SINGULAR LEVI-FLAT HYPERSURFACES IN COMPLEX PROJECTIVE SPACE INDUCED BY CURVES IN THE GRASSMANNIAN
}

\author{
JIŘÍ LEBL
}

\begin{abstract}
Let $H \subset \mathbb{P}^{n}$ be a real-analytic subvariety of codimension one induced by a real-analytic curve in the Grassmannian $G(n+1, n)$. Assuming $H$ has a global defining function, we prove $H$ is Levi-flat, the closure of its smooth points of top dimension is a union of complex hyperplanes, and its singular set is either of dimension $2 n-2$ or dimension $2 n-4$. If the singular set is of dimension $2 n-4$, then we show the hypersurface is algebraic and the Levi-foliation extends to a singular holomorphic foliation of $\mathbb{P}^{n}$ with a meromorphic (rational of degree 1) first integral. In this case, $H$ is in some sense simply a complex cone over an algebraic curve in $\mathbb{P}^{1}$. Similarly if $H$ has a degenerate singularity, then $H$ is also algebraic. If the dimension of the singular set is $2 n-2$ and is nondegenerate, we show by construction that the hypersurface need not be algebraic nor semialgebraic. We construct a Levi-flat real-analytic subvariety in $\mathbb{P}^{2}$ of real codimension 1 with compact leaves that is not contained in any proper real-algebraic subvariety of $\mathbb{P}^{2}$. Therefore a straightforward analogue of Chow's theorem for Levi-flat hypersurfaces does not hold.
\end{abstract}

\section{INTRODUCTION}

Let $\mathbb{P}^{n}$ be the $n$-dimensional complex projective space, the space of complex lines through the origin in $\mathbb{C}^{n+1}$. Chow's theorem [7] tells us that any complex analytic subvariety of $\mathbb{P}^{n}$ is algebraic. We naturally ask if Chow's theorem extends to singular Levi-flat hypersurfaces. A Levi-flat hypersurface is, after all, essentially a family of complex submanifolds. In $\mathbb{P}^{1}$ the answer is trivially no. In $\mathbb{P}^{n}, n \geq 2$, the question is significantly more difficult. To this end we study real-analytic subvarieties induced by a curve in the Grassmannian $G(n+1, n)$, the space of complex hyperplanes in $\mathbb{P}^{n}$. Such subvarieties are possibly the simplest examples of singular Levi-flat hypersurfaces in $\mathbb{P}^{n}$. While for certain hypersurfaces of this type Chow's theorem holds, we show it does not hold in general.

A smooth real hypersurface is Levi-flat if it is pseudoconvex from both sides. When the hypersurface is real-analytic, then there exist local holomorphic coordinates $z$ such that the hypersurface is given by

$$
\operatorname{Im} z_{1}=0 .
$$

For any fixed $t \in \mathbb{R},\left\{z_{1}=t\right\}$ defines a complex hypersurface, and these hypersurfaces give a real-analytic foliation called the Levi-foliation.

Let $H \subset \mathbb{P}^{n}$ be a real-analytic subvariety of codimension 1 (dimension $2 n-1$ ). Let $H_{s}$ be the singular locus of $H$, that is the set of points where $H$ fails to be a real-analytic submanifold. Let $H^{*}$ be the set of points near which $H$ is a real-analytic submanifold of dimension $2 n-1$. We say $H$ is Levi-flat if $H^{*}$ is Levi-flat. When a leaf of the Levi-foliation is closed in $H^{*}$, we consider the closure in $\mathbb{P}^{n}$ of this leaf as the leaf itself, and we say the

Date: December 17, 2014.

The first author was in part supported by NSF grant DMS-1362337 and Oklahoma State University's DIG and ASR grants. 
leaf is compact. A compact leaf is a complex analytic subvariety of $\mathbb{P}^{n}$, and hence by Chow's theorem algebraic. In our case, leaves will be complex hyperplanes in $\mathbb{P}^{n}$.

Singular Levi-flat hypersurfaces have been the subject of much recent interest. Such hypersurfaces were first studied by Bedford [1] and Burns and Gong [5]. The study of the singular set is related to the problem of extending the Levi-foliation to a singular holomorphic foliation of a neighbourhood, or more generally to a holomorphic $k$-web, see Brunella [3, 4], Fernández-Pérez [9], Cerveau and Lins Neto [6], Shafikov and Sukhov [16], and the author [13]. A singular holomorphic foliation of codimension one is given locally by an integrable holomorphic one-form $\omega$, that is, $d \omega \wedge \omega=0$. The integral manifolds are the leaves of the foliation, and the set where $\omega$ vanishes is the singular set of the foliation. A holomorphic foliation extends the Levi-foliation if the leaves of the two foliations agree as germs at points that are regular points for both the foliation and $H$.

In projective space, these hypersurfaces have been studied by the author [12], FernándezPérez [10], and Fernández-Pérez, Mol and Rosas [11]. A related question is the nonexistence of smooth Levi-flat hypersurfaces in $\mathbb{P}^{n}$, a line of research that initiated by Lins Neto [15] in the real-analytic case for $n \geq 3$. The technique of Lins Neto centers on extending the foliation to all of $\mathbb{P}^{n}$.

The first instinct to construct a singular Levi-flat hypersurface in $\mathbb{P}^{n}$ is to take a real one dimensional curve in the Grassmannian $G(n+1, n)$, which is the set of complex hyperplanes in $\mathbb{P}^{n}$. If the induced set is a subvariety, we have a Levi-flat hypersurface. We will construct such an example in $\mathbb{P}^{2}$. One might think it should be easy to take a nonalgebraic real curve and obtain a nonalgebraic hypersurface $H \subset \mathbb{P}^{n}$, but in general the induced set $H$ fails to be a subvariety or even a semianalytic set.

Let us first characterize those hypersurfaces induced by a curve in the Grassmannian. Let $C \subset G(n+1, n)$. We say a real-analytic subvariety $H \subset \mathbb{P}^{n}$ is induced by $C$ if $H$ is the smallest closed real-analytic subvariety of $\mathbb{P}^{n}$ containing all the complex hyperplanes corresponding to points in $C$. It follows from Proposition 2.1 that the set of complex hyperplanes in $H$ is a closed subvariety of $G(n+1, n)$. We therefore lose no generality in assuming $C$ is a closed real-analytic subvariety of dimension 1 (a curve).

Theorem 1.1. Suppose $H \subset \mathbb{P}^{n}$ is a real-analytic subvariety of real codimension one induced by an irreducible real-analytic curve in $G(n+1, n)$. Suppose there exists a nontrivial realanalytic function $r$ defined in a neighbourhood $V$ of $H$, such that $r$ vanishes on $H$. Then

(i) $H$ is Levi-flat,

(ii) the topological closure $\overline{H^{*}}$ is a union of complex hyperplanes ${ }^{1}$,

(iii) $\operatorname{dim} H_{s}=2 n-2$ or $\operatorname{dim} H_{s}=2 n-4$.

If $\operatorname{dim} H_{s}=2 n-4$, then there exist homogeneous coordinates $\left[z_{0}, \ldots, z_{n}\right]$ such that

(iv) the set $H_{s}$ is given by $z_{1}=z_{2}=0$,

(v) $H$ is defined by a real bihomogeneous polynomial equation $\rho\left(z_{1}, z_{2}, \bar{z}_{1}, \bar{z}_{2}\right)=0$,

(vi) the holomorphic foliation given by $z_{2} d z_{1}-z_{1} d z_{2}$ extends the Levi-foliation of $H^{*}$.

A smooth hypersurface is Levi-flat if and only if it contains a complex hypersurface through every point. Therefore, (i) follows directly from (ii). The germ of the complex hypersurface through any point is unique, and hence (ii) says that the only complex hypersurfaces in $H^{*}$ are complex hyperplanes.

\footnotetext{
$\overline{{ }^{1} \text { Note that } \overline{H^{*}}}$ may be a proper subset of $H$ even if $H$ is irreducible. See Example 1.3
} 
A real-analytic subvariety that has a global defining real-analytic function is called $\mathbb{C}$ analytic. Equivalently such a subvariety is the real trace of a complex analytic subvariety of a neighbourhood in the complexification. See [8] for more information. For example, an algebraic real-analytic subvariety of $\mathbb{P}^{n}$ is $\mathbb{C}$-analytic: Suppose $H \subset \mathbb{P}^{n}$ is given in homogeneous coordinates as the zero set of a degree $(k, k)$ bihomogeneous polynomial $r(z, \bar{z})$, that is, $r(\lambda z, \overline{\lambda z})=|\lambda|^{2 k} r(z, \bar{z})$. The expression $\frac{r(z, \bar{z})}{\|z\|^{2 k}}$ defines a real-analytic function on all of $\mathbb{P}^{n}$, whose zero set is $H$. The theorem therefore holds for all real algebraic Levi-flat hypersurfaces, though there exist many non-algebraic $\mathbb{C}$-analytic subvarieties in $\mathbb{P}^{n}$ as well. With the techniques used in the proof, it is impossible to avoid the condition that $H$ is contained in a $\mathbb{C}$-analytic set. It is, however, a natural requirement as a non- $\mathbb{C}$-analytic subvariety may be several unrelated subvarieties glued together, see e.g. [14].

The function $\frac{z_{1}}{z_{2}}$ is the rational first integral of the foliation given by $z_{2} d z_{1}-z_{1} d z_{2}$. In case $\operatorname{dim} H_{s}=2 n-4$, in the $\left(z_{1}, z_{2}\right)$-space, $H$ is a complex cone over a nonsingular algebraic curve. Note that homogeneous coordinates on $\mathbb{P}^{n}$ are given up to an automorphism of $\mathbb{P}^{n}$, i.e. an invertible linear map of $\mathbb{C}^{n+1}$.

If $H$ is induced by a curve in the Grassmanian, we say a point $p \in H_{s}$ is degenerate if infinitely many complex hyperplanes in $H$ pass through $p$. If $\operatorname{dim} H_{s}=2 n-4$, then every point in $H_{s}$ is degenerate. Our next result says that if $H$ has a degenerate singularity then $H$ arises from an algebraic surface in lower dimension. A curve in $\mathbb{P}^{1}$ has no degenerate singularities.

Theorem 1.2. Suppose $H \subset \mathbb{P}^{n}$ is a real-analytic subvariety of real codimension one induced by an irreducible real-analytic curve in $G(n+1, n)$, further suppose $H_{s}$ contains a degenerate singular point. Then there exists an integer $1 \leq \ell<n$, homogeneous coordinates $\left[z_{0}, \ldots, z_{n}\right]$, and a bihomogeneous real polynomial $\rho\left(z_{0}, \ldots, z_{\ell}, \bar{z}_{0}, \ldots, \bar{z}_{\ell}\right)$, such that

(i) $H$ is the zero set of $\rho$ (in particular, $H$ is algebraic),

(ii) $\rho$ defines a Levi-flat algebraic hypersurface $X \subset \mathbb{P}^{\ell}$ with no degenerate singularities induced by a curve in $G(\ell+1, \ell)$.

Theorem 1.2 implies that a nonalgebraic $H$ has no degenerate singularities. The assumption that the curve in $G(n+1, n)$ is irreducible is equivalent to assuming $H$ is induced by a nonsingular germ of a curve in $G(n+1, n)$. The theorem also justifies our claim that a subvariety in $G(n+1, n)$ does not necessarily induce a subvariety in $\mathbb{P}^{n}$. If we take a nonalgebraic curve in $G(n+1, n)$ where the planes all go through a single point, then there cannot be a proper subvariety of $\mathbb{P}^{n}$ containing all the hyperplanes as Proposition 2.1 would imply it be nonalgebraic, and that contradicts the theorem.

Example 1.3 (Whitney umbrella with complex lines). Let us discuss a crucial algebraic example in $\mathbb{C}^{2}$ (and hence in $\mathbb{P}^{2}$ ) showing that $H$ itself may not be a union of complex hyperplanes, and that the Levi-foliation need not extend. Let $(z, w) \in \mathbb{C}^{2}$ be our coordinates and write $z=x+i y$ and $w=s+i t$. Consider the hypersurface $H$ given by

$$
s y^{2}-t x y-t^{2}=0 \text {. }
$$

The hypersurface is Levi-flat with the nonsingular hypersurface part foliated by the complex lines given by $w=c z+c^{2}$, where $c \in \mathbb{R}$. Indeed, setting $z=\xi$ and $w=c \xi+c^{2}$ gives a $\overline{H^{*}}$ as an image of $\mathbb{C} \times \mathbb{R}$. This irreducible hypersurface has a lower-dimensional totally-real component given by $\{t=0, y=0\}$, "half of it" (for $s$ sufficiently negative) sticking out of the hypersurface as the "umbrella handle." In the complement of $\{y=0\}$, the hypersurface 
$H$ is a graph: $s=\frac{t x y+t^{2}}{y^{2}}$ or $s=x\left(\frac{t}{y}\right)+\left(\frac{t}{y}\right)^{2}$. The set $\left\{t=0, y=0, s<\frac{-x^{2}}{4}\right\}$ is not in the closure of $H^{*}$. Therefore, not all of $H$ is a union of complex lines; only the closure $\overline{H^{*}}$ is such a of complex lines, as claimed in Theorem 1.1. Notice that when $x=0$, we obtain the standard Whitney umbrella in $\mathbb{R}^{3}[17]$.

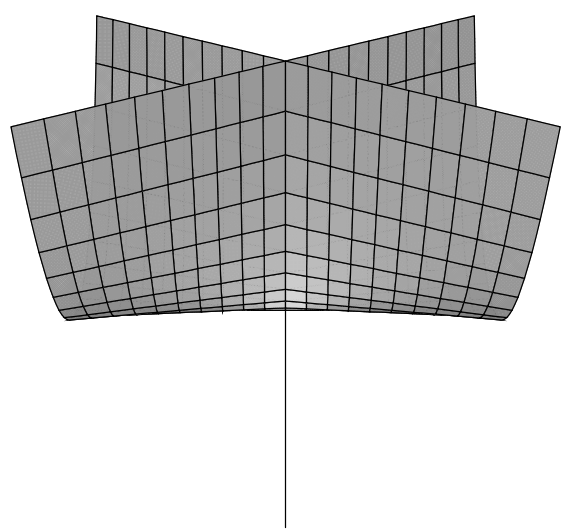

Figure 1. The Whitney Umbrella in $\mathbb{R}^{3}$.

The singular set $H_{s}=\left\{t=0, y=0, s \geq \frac{-x^{2}}{4}\right\}$ is of real dimension 2 , and it is a generic manifold at points arbitrarily near the origin. Therefore, the Levi-foliation does not extend as a singular holomorphic foliation of a neighbourhood of the origin. The Levi-foliation does extend as a holomorphic 2-web given by $d w^{2}+z d z d w-w d z^{2}=0$. Its multivalued first integral is obtained by applying the quadratic formula to $w=c z+c^{2}$. See $[3,16]$.

See [12] for an example of an umbrella-type subvariety composed of complex lines where the "umbrella handle" is a complex line.

Example 1.4. A simple but illustrative example is the hypersurface $H$ in $\mathbb{P}^{2}$ given in homogeneous coordinates $\left[z_{0}, z_{1}, z_{2}\right]$ as

$$
\left|z_{1}\right|^{2}=\left|z_{2}\right|^{2}
$$

The singularity is the set $\left\{z_{1}=z_{2}=0\right\}$; that is, a single point in $\mathbb{P}^{2}$, which is a degenerate singularity. The singular holomorphic foliation extending the Levi-foliation is given by the one-form $z_{2} d z_{1}-z_{1} d z_{2}$ as claimed, and $H$ is the cone over a circle in $\mathbb{P}^{1}$.

One of the primary motivations of this paper is to find a nonalgebraic singular Levi-flat hypersurface in projective space. Previously in [12], the author proved an analogue of Chow's theorem for Levi-flat hypersurfaces under extra hypotheses: A Levi-flat subvariety whose Levi-foliation extends as a singular holomorphic foliation at each point and has infinitely many compact leaves is contained in a real-algebraic subvariety. It should be noted that a real-algebraic Levi-flat automatically has compact leaves.

Theorem 1.5. There exists a real-analytic singular Levi-flat hypersurface $H \subset \mathbb{P}^{2}$ with all leaves complex hyperplanes, such that $H^{*}$ (and hence $H$ ) is not contained in any proper real-algebraic subvariety of $\mathbb{P}^{2}$.

Therefore, Chow's theorem does not hold for Levi-flat hypersurfaces without extra assumptions. Also, the hypotheses in [12] are not unnecessary; the nonalgebraic hypersurface 
we construct has compact leaves, but the Levi-foliation does not extend at certain points of its singular set.

We further construct an example of a real-analytic singular Levi-flat hypersurface $H$ that is semialgebraic, but not algebraic. That is, we construct an irreducible algebraic Levi-flat hypersurface globally reducible into two components as a real-analytic subvariety.

The author would like to acknowledge Xianghong Gong for suggesting the original question. The author would like to thank Arturo Fernández-Pérez for many discussions on the topic, and several very useful suggestions and corrections to the manuscript.

\section{Real-AnAlytic SUbVARIETIEs IndUCED By CURVES In the Grassmannian}

A real-analytic subvariety $H \subset \mathbb{P}^{n}$ is algebraic if it is the zero set of a bihomogeneous polynomial. A set is semialgebraic, if it is defined by a finite set of algebraic equalities and inequalities. A real-analytic subvariety can be semialgebraic, but not algebraic; a realanalytic algebraic subvariety can be irreducible as an algebraic subvariety, but reducible as a real-analytic subvariety.

Let us fix some notation. Let $\sigma: \mathbb{C}^{n+1} \backslash\{0\} \rightarrow \mathbb{P}^{n}$ be the natural projection. If $X$ is a real-analytic subvariety of $\mathbb{P}^{n}$, then let $\tau(X) \subset \mathbb{C}^{n+1}$ to be the set of points $z \in \mathbb{C}^{n+1}$ such that $\sigma(z) \in X$ or $z=0$. We say $Y \subset \mathbb{C}^{n+1}$ is a complex cone if $z \in Y$ implies $\lambda z \in Y$ for all $\lambda \in \mathbb{C}$. For example, $\tau(X)$ is a complex cone. If $Y \subset \mathbb{C}^{n+1}$ is a complex cone we write $\sigma(Y)$ for the induced set in $\mathbb{P}^{n}$.

While $X \subset \mathbb{P}^{n}$ may be a real-analytic subvariety, the set $\tau(X)$ need not be a subvariety. The problem is at the origin, clearly $\tau(X) \backslash\{0\}$ is a subvariety of $\mathbb{C}^{n+1} \backslash\{0\}$. This is precisely where the proof of Chow's theorem breaks down in the general real-analytic case. Via the standard proof of Chow's theorem (see e.g. [12]), if $\tau(X)$ is a variety, then $X$ is algebraic. If $\tau(X)$ is semianalytic (in particular contained in a real-analytic subvariety of the same dimension), then $X$ is semialgebraic.

Before we do anything else, we prove the following proposition promised in the introduction. It means that every subvariety $H \subset \mathbb{P}^{n}$ induces a subvariety (possibly empty) in $G(n+1, n)$ of hyperplanes contained in $H$. Therefore, from now on we can assume the curve that induces our hypersurfaces is a closed real-analytic subvariety of dimension 1.

Proposition 2.1. Suppose $X \subset \mathbb{P}^{n}$ is a proper real-analytic subvariety. Then the set $C \subset$ $G(n+1, n)$ of complex hyperplanes contained in $X$ is a real-analytic subvariety of $G(n+1, n)$ of dimension 1 or less. Furthermore, if $X$ is algebraic, then $C$ is algebraic.

Proof. Suppose $L$ is a hyperplane in $G(n+1, n)$ passing through a point $p \in \mathbb{P}^{n}$. Without loss of generality, let $p$ be the origin in inhomogeneoous coordinates given by $z_{0}=1$, and suppose $L$ is given by $z_{1}=0$. The hyperplanes in $G(n+1, n)$ in a neighbourhood of $L$ are given by the equation

$$
z_{1}=a_{0}+a_{2} z_{2}+\cdots+a_{n} z_{n}
$$

for $a_{j}$ small. The corresponding hyperplane is contained in $X$ if as a function of $z_{2}, \ldots, z_{n}$ and $\bar{z}_{2}, \ldots, \bar{z}_{n}$,

$$
\rho\left(a_{0}+a_{2} z_{2}+\cdots+a_{n} z_{n}, z_{2}, \ldots, z_{n}, \overline{a_{0}+a_{2} z_{2}+\cdots+a_{n} z_{n}}, \bar{z}_{2}, \ldots, \bar{z}_{n}\right) \equiv 0
$$

for all real-analytic $\rho$ that vanish on $X$ near $p$. Expanding as a series in $z_{2}, \ldots, z_{n}, \bar{z}_{2}, \ldots, \bar{z}_{n}$

$$
\sum c_{j_{2}, \ldots, j_{n}, k_{2}, \ldots, k_{n}}\left(a_{0}, a_{2}, \ldots, a_{n}, \bar{a}_{0}, \bar{a}_{2}, \ldots, \bar{a}_{n}\right) z_{2}^{j_{2}} \cdots z_{n}^{j_{n}} \bar{z}_{2}^{k_{2}} \cdots \bar{z}_{n}^{k_{n}}
$$


and setting its coefficients to 0 , we obtain real-analytic equations for $a_{0}, a_{2}, \ldots, a_{n}$. The result follows.

The dimension claim follows easily; take a two-dimensional submanifold of $C(n+1, n)$ and note that the corresponding hyperplanes fill an open subset of $\mathbb{P}^{n}$. Finally if $X$ is algebraic then take only polynomial $\rho$ above and the equations giving $C$ are polynomial and hence $C$ is algebraic.

The proposition has the following corollary. If $H$ is induced by an irreducible curve $C \subset G(n+1, n)$, then $H$ is irreducible. If $H$ was reducible into two smaller components, at least one of them contains all the complex hyperplanes from $C$.

We have the following special case of a well-known and easy observation.

Proposition 2.2. Suppose $H \subset \mathbb{P}^{n}$ is a real-analytic subvariety of real codimension one induced by a real-analytic curve in $G(n+1, n)$. Then $2 n-4 \leq \operatorname{dim} H_{s} \leq 2 n-2$.

Proof. A smooth hypersurface cannot contain two distinct complex hyperplanes through a point. Thus any point where two hyperplanes meet must be singular. Any two complex hyperplanes in $\mathbb{P}^{n}$ meet along a set of complex dimension $n-2$ or real dimension $2 n-4$.

Before getting into the details of the proof of Theorem 1.1 let us start with complexification of $\mathbb{P}^{n}$ as a real-analytic manifold. We write $\mathcal{M}$ for the obvious complexification of $\mathbb{P}^{n}$; let $z=\left(z_{0}, \ldots, z_{n}\right)$ be the homogeneous coordinates, then we let $w=\bar{z}$ be a new variable and we have $\mathcal{M} \cong \mathbb{P}^{n} \times \mathbb{P}^{n}$. Then $\mathbb{P}^{n}$ is imbedded as a real-analytic totally-real submanifold via $z \mapsto(z, \bar{z})$. It is a classic result of Grauert (see also [8]) that a real-analytic (paracompact) manifold has a fundamental system of Stein neighbourhoods in its complexification. This result is not completely necessary in the proof below, but it simplifies the proof. This fact may seem a little counterintuitive about $\mathbb{P}^{n}$, but recall we are talking about $\mathbb{P}^{n}$ only as a real-analytic submanifold, ignoring the complex structure.

The first two claims of Theorem 1.1 follow from the following lemma.

Lemma 2.3. Suppose $H \subset \mathbb{P}^{n}$ is a real-analytic subvariety of real codimension 1, induced by an irreducible real-analytic curve in $G(n+1, n)$. Suppose there exists a nontrivial realanalytic function $r$ defined in a neighbourhood $V$ of $H$, such that $r$ vanishes on $H$. Then $\overline{H^{*}}$ is a union of complex hyperplanes.

Proof. Take a small smooth piece of the curve. In homogeneous coordinates on $\mathbb{P}^{n}$, we write the corresponding planes (after perhaps reordering coordinates) as

$$
z_{0}=a_{1}(t) z_{1}+\cdots+a_{n}(t) z_{n}
$$

where $a_{j}(0)=0$ for all $j$, but $a_{k}^{\prime}(0) \neq 0$ for at least one $k$. By implicit function theorem, for most points on the leaf $z_{0}=0$, we can solve for $t$, and hence near such points the union of the planes is a $2 n-1$ dimensional real submanifold. In particular, there is a point $q \in H^{*}$, a neighbourhood of which in $H^{*}$ is locally a union of complex hypersurfaces.

We complexify as above and consider $\mathbb{P}^{n} \subset \mathcal{M}$ as a totally-real subset. Let $r$ and $V$ be as given. This real-analytic function complexifies to some neighbourhood $U$ of $H$ in the complexification $\mathcal{M}$. As $V$ is a real-analytic submanifold it has a fundamental system of Stein neighbourhoods in $\mathcal{M}$ and we can therefore assume that $U$ is Stein. The zero set of $r$ is a complex analytic subvariety $\mathcal{X}$ of $U$ such that $H \subset \mathcal{X} \cap \mathbb{P}^{n}$. Assume $\mathcal{X}$ is irreducible (as $H$ is irreducible). As $\mathcal{X}$ is complex analytic in a Stein domain, it is coherent. We can assume the complexified $r$ to be the defining function for $\mathcal{X}$ such that for every $p \in \mathcal{X}$, the 
function $r$ generates the ideal $I((\mathcal{X}, p))$ of germs at $p$ of holomorphic functions vanishing on the germ $(\mathcal{X}, p)$. In particular, the derivative $d r$ vanishes only on the singular locus of $\mathcal{X}$. The singular locus of $\mathcal{X}$ intersected with $H$ is a proper subvariety of $H$ of dimension less than $2 n-1$. Hence $d r$ vanishes on a subvariety of $H$ of dimension less than $2 n-1$.

Let $\left(H^{*}\right)^{\prime}=\left\{z \in H^{*}: d r(z, \bar{z}) \neq 0\right\}$. The set $\left(H^{*}\right)^{\prime}$ is dense and open in $H^{*}$. Therefore we assume that $q \in\left(H^{*}\right)^{\prime}$. The real form $\theta=i(\partial r-\bar{\partial} r)$ defines the space $T_{q}^{(1,0)} H \oplus T_{q}^{(0,1)} H$. This form complexifies and also defines a (complex) codimension 1 subspace $\mathcal{T}$ on the tangent space of $\mathcal{X}$ at smooth points, which agrees with $T_{p}^{(1,0)} H \oplus T_{p}^{(0,1)} H$ at all $p \in\left(H^{*}\right)^{\prime}$. As a form on $H^{*}, \theta$ is integrable near $q$ (as $H$ is Levi-flat there), then the complexified $\theta$ is integrable on the regular points of $\mathcal{X}$ and hence on all of $\left(H^{*}\right)^{\prime}$.

So $H^{*}$ is Levi-flat, we now need to show that the leaves are locally complex hyperplanes. It is again enough to do so on a dense subset of $H^{*}$. To make things simpler let us move to inhomogeneous coordinates with $z_{0}=1$, and note that $H^{*} \cap\left\{z_{0}=1\right\}$ is dense in $H^{*}$. Compute the Hessian (the full real Hessian) $H r$ of $r$, and note that it vanishes on $T_{q}^{(1,0)} H \oplus T_{q}^{(0,1)} H$ for the point $q \in\left(H^{*}\right)^{\prime}$ where $H$ is a union of complex hyperplanes. The function $H r$ also complexifies (the complexification is the Hessian of the complexified $r$ ). The complexified $H r$ is identically zero on the vectors in $\mathcal{T}$ at all points in $\mathcal{X}$ near $(q, \bar{q})$. By identity $H r$ vanishes on all the vectors in $\mathcal{T}$ at all the regular points of $\mathcal{X}$ for which neither $z_{0}=0$ nor $w_{0}=0$ (the set of regular points of $\mathcal{X}$ is connected).

Then at all points $p \in\left(H^{*}\right)^{\prime} \backslash\left\{z_{0}=0\right\}$, the Hessian $H r$ also vanishes on $T_{p}^{(1,0)} H \oplus T_{p}^{(0,1)} H$. The leaves of the Levi-foliation near $p$ must then be complex hyperplanes (no curvature). That means the leaves near all points of $H^{*}$ are complex hyperplanes.

Take a complex hyperplane $L$ such that an open neighbourhood of it is in $H^{*}$. Clearly $L \subset H$. What is left to show is that $L$ is in the closure of $H^{*}$. Suppose we pick a point in $H^{*}$ near which the leaves are complex hyperplanes. At such a point, taking a nonsingular curve $\gamma$ in $H^{*}$ transverse to the leaves, we obtain that the leaves nearby clearly form a small nonsingular curve in $G(n+1, n)$. By the argument in the beginning of the proof, on a open dense subset of $L$, the set $L$ is part of a smooth $2 n-1$ dimensional manifold. In other words, the leaf is in the closure $\overline{H^{*}}$.

The limit set of complex planes is a union of complex planes, and hence $\overline{H^{*}}$ is a union of complex planes.

\section{Degenerate singularities}

To prove Theorem 1.2, it is enough to prove the following lemma. The lemma will also be useful in the proof of Theorem 1.1 .

Lemma 3.1. Suppose $H \subset \mathbb{P}^{n}$ is a real-analytic subvariety of real codimension one induced by an irreducible real-analytic curve in $G(n+1, n)$ and suppose $H_{s}$ has a degenerate singularity. Then there exist homogeneous coordinates $\left[z_{0}, \ldots, z_{n}\right]$ such that $H$ has a real polynomial defining function depending on $z_{0}$ through $z_{n-1}$ only.

To obtain Theorem 1.2, that is, to obtain an $X$ with no nondegenerate singularities, we repeatedly apply the lemma, noting a curve in $\mathbb{P}^{1}$ cannot have degenerate singularities. Then for the $\ell$ in the theorem there exists an $X \subset \mathbb{P}^{\ell}$ defined by the polynomial in $z_{0}$ through $z_{\ell}$, such that $\tau(H)=\tau(X) \times \mathbb{C}^{n-\ell}$. To prove the lemma, it is enough to show that in inhomogeneous coordinates where the origin is the degenerate singularity, $H$ is a complex 
cone defined by a bihomogeneous polynomial, since then we have eliminated one variable from the defining equation and we have shown algebraicity.

Proof. Let us work in inhomogeneous coordinates defined by $z_{0}=1$, and suppose the degenerate point is the origin. We proceed as in the standard proof of Chow's theorem. Let $\rho$ be a real-analytic defining function for $H$ near the origin. Decompose $\rho$ into bihomogeneous components as

$$
\rho(z, \bar{z})=\sum_{j k} \rho_{j k}(z, \bar{z}) .
$$

We have infinitely many complex hyperplanes passing through the origin.

Let $V$ be the union of the hyperplanes through the origin contained in $H$ near the origin. If $z$ in $V$, then $\lambda z \in V$ for all $\lambda \in \mathbb{C}$, and so

$$
0=\rho(\lambda z, \bar{\lambda} \bar{z})=\sum_{j k} \lambda^{j} \bar{\lambda}^{k} \rho_{j k}(z, \bar{z}) .
$$

So for each $j$ and $k, \rho_{j k}(z, \bar{z})=0$ for $z \in V$. Let $\widetilde{H}$ be the algebraic variety defined by $\rho_{j k}(z, \bar{z})=0$ for all $j$ and $k$. Clearly as germs at the origin $(\widetilde{H}, 0) \subset(H, 0)$. Furthermore $\widetilde{H}$ must be of real dimension $2 n-1$, and $\widetilde{H}$ is complex cone as it is defined by bihomogeneous polynomials.

If $z \in \widetilde{H}$, then $\lambda z \in \widetilde{H}$ for all $\lambda \in \mathbb{C}$. For small $\lambda, \lambda z \in H$ as $\widetilde{H} \subset H$ as germs at the origin. But as $H$ is a subvariety we have that $\lambda z \in H$ for all $\lambda \in \mathbb{C}$, in particular if $\lambda=1$, and so $z \in H$. We have shown that $\widetilde{H} \subset H$. As the curve of complex hyperplanes in $H$ is irreducible and $H$ is the smallest subvariety contining those planes $\widetilde{H}=H$. Hence $H$ is algebraic.

Finally, as $H$ is a complex cone in $\mathbb{C}^{n}$ it induces a subvariety $X \subset \mathbb{P}^{n-1}$. In the right coordinates $\tau(H)=\tau(X) \times \mathbb{C}$. The lemma follows.

We remark the lemma implies that to study hypersurfaces induced by curves in the Grassmannian, it is enough to study those that have no degenerate singularities.

\section{EXTENDing THE FOLIATION WHEN THE SINGULARITY IS SMALL}

Given a subvariety $C \subset G(n+1, n)$, let $S \subset \mathbb{P}^{n}$ be the set of points where two or more complex hyperplanes in $C$ intersect. The set $S$ is given in homogeneous coordinates by $\left\{z \in \mathbb{C}^{n+1}: z \cdot a=z \cdot b=0,[a] \neq[b],[a] \in C,[b] \in C\right\}$. That is a subanalytic set, see e.g. [2].

Lemma 4.1. Let $C \subset G(n+1, n), n \geq 2$, be an irreducible real-analytic subvariety of real dimension one. Let $S \subset \mathbb{P}^{n}$ be the set of points where two or more complex hyperplanes in $C$ intersect. If $\operatorname{dim}_{\mathbb{R}} S \leq 2 n-3$, then after an automorphism of $\mathbb{P}^{n}$, every hyperplane in $C$ is given by an equation of the form

$$
0=a_{1} z_{1}+a_{2} z_{2}
$$

and the set $S$ is given by the equations $z_{1}=z_{2}=0$. In particular, $\operatorname{dim}_{\mathbb{R}} S=2 n-4$.

Proof. Let $z_{0}, z_{1}, \ldots, z_{n}$ be the homogeneous coordinates on $\mathbb{P}^{n}$. An automorphism of $\mathbb{P}^{n}$ is an invertible linear transformation in $n+1$ variables. Pick two distinct smooth points on $C$. Work in inhomogeneous coordinates given by $z_{0}=1$. Without loss of generality, after 
a change of coordinates, assume the two hyperplanes are given by $z_{1}=0$ and $z_{2}=0$. We parametrize the curve $C$ near the two points and hence the corresponding hyperplanes as

$$
\begin{aligned}
& z_{1}=a_{0}(s)+a_{2}(s) z_{2}+a_{3}(s) z_{3}+\cdots+a_{n}(s) z_{n}, \\
& z_{2}=b_{0}(t)+b_{1}(t) z_{1}+b_{3}(t) z_{3}+\cdots+b_{n}(t) z_{n} .
\end{aligned}
$$

The functions $a_{k}$ and $b_{k}$ are real-analytic functions of one real variable defined near the origin and vanishing at 0 .

The hypotheses of the lemma are that the intersection of these two families of planes is of dimension less than or equal to $2 n-3$. Furthermore we can assume that as a vector valued function, the $a$ and the $b$ is non-constant. That is, not all $a_{k}$ are identically zero, and similarly not all $b_{k}$ are identically zero. We will show that $a_{0}$ and $a_{3}, \ldots, a_{n}$ must be identically zero, and $b_{0}$ and $b_{3}, \ldots, b_{n}$ must be identically zero. That is, only $a_{2}$ and $b_{1}$ are allowed to vary (they must vary in fact). Once this is proved, the lemma holds.

Let us suppose for contradiction the above assertion is not true. In particular, after a linear change of variables in $z_{0}, z_{3}, \ldots, z_{n}$, assume $b_{0}$ and $a_{0}$ are not both identically zero. By symmetry, assume it is $a_{0}$ that is not identically zero. If $b_{0}$ is identically zero after a generic linear change of variables as above, then $b_{3}$ through $b_{n}$ are identically zero as well and in that case $b_{1}$ is not identically zero.

Solving for $z_{1}$ and $z_{2}$, we obtain a piece of $S$ parametrized by $s, t$, and $z_{3}, \ldots, z_{n}$ as

$$
\begin{aligned}
& z_{1}=\frac{a_{0}(s)+a_{2}(s)\left(b_{0}(t)+b_{3}(t) z_{3}+\cdots+b_{n}(t) z_{n}\right)+a_{3}(s) z_{3}+\cdots+a_{n}(s) z_{n}}{1-a_{2}(s) b_{1}(t)}, \\
& z_{2}=\frac{b_{0}(t)+b_{1}(t)\left(a_{0}(s)+a_{3}(s) z_{3}+\cdots+a_{n}(s) z_{n}\right)+b_{3}(t) z_{3}+\cdots+b_{n}(t) z_{n}}{1-a_{2}(s) b_{1}(t)} .
\end{aligned}
$$

where $z_{3}, \ldots, z_{n}$ simply parametrize themselves. As dimension of the image is less than or equal to $2 n-3$, it must be true when we set $z_{3}$ through $z_{n}$ to zero, the rank of the resulting mapping (rank of the derivative)

$$
\begin{aligned}
& z_{1}=\frac{a_{0}(s)+a_{2}(s) b_{0}(t)}{1-a_{2}(s) b_{1}(t)}, \\
& z_{2}=\frac{b_{0}(t)+b_{1}(t) a_{0}(s)}{1-a_{2}(s) b_{1}(t)},
\end{aligned}
$$

must be of rank strictly less than 2 at all points. Clearly this is possible if both $a_{0}$ and $b_{0}$ are both identically 0 , however, we supposed for contradiction $a_{0}$ is not identically zero.

If neither $a_{0}$ nor $b_{0}$ is identically zero, then the mapping is a finite map. That is, solving $a_{0}(s)+a_{2}(s) b_{0}(t)=0$, and $b_{0}(t)+b_{1}(t) a_{0}(s)=0$ for $s$ and $t$ near zero implies that both $a_{0}$ and $b_{0}$ must be zero. A finite map has generically full rank, that is 2 , which contradicts our assumption on rank.

The final case to check is when $b_{0}$ is identically zero. The mapping becomes $\frac{a_{0}(s)}{1-a_{2}(s) b_{1}(t)}$ and $\frac{b_{1}(t) a_{0}(s)}{1-a_{2}(s) b_{1}(t)}$, and that is easily seen to have derivative of rank 2 for generic points $s, t$ near the origin if $b_{1}$ is not identically zero. By assumption, $b_{1}$ is not identically zero.

By irreducibility of $C$ we find that if $S$ is contained in all complex hyperplanes in some open set in $C$, then $S$ is contained in all complex hyperplanes in $C$.

The following lemma proves the remainder of the claims in Theorem 1.1 . 
Lemma 4.2. Suppose $H \subset \mathbb{P}^{n}$ is a real-analytic subvariety of real codimension one induced by an irreducible real-analytic curve in $G(n+1, n)$ and suppose $\operatorname{dim} H_{s} \leq 2 n-3$. Then after an automorphism of $\mathbb{P}^{n}$, there exist homogeneous coordinates $\left[z_{0}, \ldots, z_{n}\right]$ such that

(i) $H$ has a real polynomial defining function depending on $z_{1}$ and $z_{2}$ only.

(ii) The holomorphic foliation given by $z_{2} d z_{1}-z_{1} d z_{2}$ extends the Levi-foliation of $H^{*}$.

(iii) The set $H_{s}$ is given by $z_{1}=z_{2}=0$ and so in particular $\operatorname{dim}_{\mathbb{R}} H_{s}=2 n-4$.

Proof. Let $C \subset G(n+1, n)$ be the subvariety defined by all complex hyperplanes in $H$. Let $C_{1}$ be an irreducible one-dimensional component of $C$. Use Lemma 4.1 to find the coordinates such that all hyperplanes in $C_{1}$ are given by linear functions of $z_{1}$ and $z_{2}$. In particular all the hyperplanes pass through the origin in the inhomogeneous coordinates given by $z_{0}=1$.

The origin is a degenerate singularity and therefore by Lemma 3.1, $H$ is algebraic and defined by a bihomogeneous polynomial.

The foliation is given by the one-form $z_{2} d z_{1}-z_{1} d z_{2}$ with the meromorphic first integral $\frac{z_{1}}{z_{2}}$, as the leaves of this foliation are precisely the complex hyperplanes given by linear functions of $z_{1}$ and $z_{2}$ only. This foliation then clearly extends the Levi-foliation of $H$.

Let us now work in homogeneous coordinates $z_{0}, \ldots, z_{n}$. The cone $\tau(H)$ is an algebraic subvariety in $\mathbb{C}^{n+1}$. By fixing $z_{0}$, and $z_{3}, \ldots, z_{n}$ at a generic value we obtain a real polynomial in $z_{1}$ and $z_{2}$ that vanishes on a set of real dimension 3 in the $\left(z_{1}, z_{2}\right)$-space. Since it must vanish on the hyperplanes in $H$ it vanishes on $H$. We obtain our defining polynomial depending on $z_{1}$ and $z_{2}$ only. Hence $H$ is a complex cone over a curve in $\mathbb{P}^{1}$.

The singular set of $H$ is therefore either given by the singular set of the foliation, which is precisely $z_{1}=z_{2}=0$, or by the complex hyperplanes that correspond to the singular points of the curve in $\mathbb{P}^{1}$. That is, $H_{s}$ is a union of the set $\left\{z_{1}=z_{2}=0\right\}$ and a finite number of complex hyperplanes corresponding to the singular points of the curve. That is $H_{s}$ is a complex subvariety and hence by assumption on dimension it must be of real dimension $2 n-4$ and cannot contain any complex hyperplanes. So the curve in $\mathbb{P}^{1}$ must be nonsingular and $H_{s}=\left\{z_{1}=z_{2}=0\right\}$.

\section{The CONSTRUCTION OF A NONALGEBRAiC HYPERSURFACE}

In this section we construct a real-analytic singular Levi-flat hypersurface $H \subset \mathbb{P}^{2}$ with all leaves compact, such that $H^{*}$ is not contained in any proper real-algebraic subvariety of $\mathbb{P}^{2}$. That is, the only bihomogeneous polynomial vanishing on all of $\tau\left(H^{*}\right)$ and hence on $\tau(H)$ is identically zero. The $H$ we construct will be induced by a nonalgebraic real-analytic curve in $G(3,2)$.

Lemma 5.1. Let $X \subset \mathbb{R}^{2}$ be a connected compact real-analytic curve with no singularities. Let $\widetilde{H}$ be the complex cone defined by

$$
\widetilde{H}=\left\{\left(z_{0}, z_{1}, z_{2}\right) \in \mathbb{C}^{3}: z_{0}=z_{1} x+z_{2} y \text { where }(x, y) \in X\right\} \cup\left\{z \in \mathbb{C}^{3}: z_{1} \bar{z}_{2}=\bar{z}_{1} z_{2}\right\} .
$$

Then $\widetilde{H}$ is a subvariety in $\mathbb{C}^{3} \backslash\{0\}$. In other words the induced set $\sigma(\widetilde{H})$ is a real-analytic subvariety of $\mathbb{P}^{2}$.

Proof. Let $X \subset \mathbb{R}^{2}$ be as above. Suppose $a, b: \mathbb{R} \rightarrow X$ are two real-valued real-analytic functions of a real variable $t$ such that the image of $(a(t), b(t))$ is all of $X$. As $X$ has no singularities, we assume the derivatives $a^{\prime}(t)$ and $b^{\prime}(t)$ do not both vanish at any $t \in \mathbb{R}$. 
Define a complex cone $\widetilde{H}$ as

$$
\widetilde{H}=\left\{\left(z_{0}, z_{1}, z_{2}\right) \in \mathbb{C}^{3}: z_{0}=z_{1} x+z_{2} y \text { where }(x, y) \in X\right\} \cup\left\{z \in \mathbb{C}^{3}: z_{1} \bar{z}_{2}=\bar{z}_{1} z_{2}\right\} .
$$

Clearly $\widetilde{H}$ is a complex cone and so $H=\sigma(\widetilde{H})$ is a well-defined subset of $\mathbb{P}^{2}$. If $\widetilde{H} \backslash\{0\}$ is a real-analytic subvariety of $\mathbb{C}^{3} \backslash\{0\}$, then $H$ is a real-analytic subvariety of $\mathbb{P}^{2}$. Assuming $\widetilde{H}$ is a real-analytic subvariety away from the origin then $H$ is Levi-flat, as $\widetilde{H}$ is a union of complex hyperplanes. In fact, $H$ is induced by a nonsingular real-analytic curve in $G(3,2)$.

It is therefore left to show $\widetilde{H}$ is a subvariety away from the origin. We write

$$
\left[\begin{array}{l}
z_{0} \\
\bar{z}_{0}
\end{array}\right]=\left[\begin{array}{ll}
z_{1} & z_{2} \\
\bar{z}_{1} & \bar{z}_{2}
\end{array}\right]\left[\begin{array}{l}
x \\
y
\end{array}\right]
$$

If the matrix is invertible we solve for $x$ and $y$ in terms of $z$. Outside the set

$$
\Delta=\left\{z: z_{1} \bar{z}_{2}-\bar{z}_{1} z_{2}=0\right\}
$$

the set $\widetilde{H}$ is an image of a real-analytic submanifold under a real-analytic diffeomorphism, and therefore a subvariety.

Let us take a point on $\widetilde{H}$ where $z_{1} \bar{z}_{2}-\bar{z}_{1} z_{2}=0$. Call this point $p=\left(z_{0}^{0}, z_{1}^{0}, z_{2}^{0}\right)$. The complex values $\bar{z}_{1}^{0}$ and $\bar{z}_{2}^{0}$ lie on the same line through the origin in $\mathbb{C}$. After moving all of $\mathbb{C}^{3}$ by a diagonal unitary we assume $z_{1}^{0}$ and $z_{2}^{0}$ are real. As they are not both zero, we find a $2 \times 2$ real orthogonal matrix $U$ that takes the point $(1,0)$ to $\left(z_{1}^{0}, z_{2}^{0}\right)$. Write $A(t)=(a(t), b(t))$. Then near $p$ and outside of $\Delta$, the set $\widetilde{H}$ is the image of

$$
\left(\xi_{1}, \xi_{2}, t\right) \mapsto((U \xi \cdot A(t)), U \xi)=\left(\left(\xi \cdot U^{t} A(t)\right), U \xi\right)
$$

where $\xi_{1}, \xi_{2}$ are complex and $t$ real. Again by moving around (post-composing the above mapping) by an orthogonal mapping we assume $z_{1}^{0}=1$ and $z_{2}^{0}=0$.

We now work in inhomogeneous coordinates in $\mathbb{C}^{2}$ where we set $z_{1}=1$ and write $z_{2}=\zeta$. The set $\Delta$ is the set where $\zeta$ is real-valued. It is enough to consider the image of the map

$$
(\zeta, t) \mapsto(a(t)+\zeta b(t), \zeta)
$$

for $\zeta \in \mathbb{C}$ near the origin and $t \in \mathbb{R}$. The question is local and so it is enough to consider $t$ near the origin. We must show the image union $\Delta$ is a real-analytic subvariety.

We complexify by letting $\bar{\zeta}=\omega$ and $\bar{t}=s$, treating $t$ as a complex variable. As neither $a$ nor $b$ vanish identically, the complexified mapping (the coefficients of $a$ and $b$ are real)

$$
(\zeta, t, \omega, s) \mapsto(a(t)+\zeta b(t), \zeta, a(s)+\omega b(s), \omega)
$$

is a finite map near the origin. Hence it is a proper holomorphic mapping between two neighbourhoods of the origin. By proper mapping theorem this map takes the set $s=t$ to a complex analytic subvariety of a neighbourhood of the origin. We restrict to the "diagonal," to what this image is intersected with the original $\mathbb{C}^{2}$ before complexification:

$$
\zeta=\bar{\omega}, \quad a(t)+\zeta b(t)=\overline{a(s)+\omega b(s)} .
$$

We substitute $\zeta=\bar{\omega}$ and $s=t$ into the right hand side of the second equation: $\overline{a(s)+\omega b(s)}=$ $a(\bar{t})+z b(\bar{t})$. So

$$
(a(t)-a(\bar{t}))+\zeta(b(t)-b(\bar{t}))=0 .
$$

As $a(t)-a(\bar{t})=a(t)-\overline{a(t)}$ and $b(t)-b(\bar{t})=b(t)-\overline{b(t)}$ are purely imaginary for all $t \in \mathbb{C}$, either $a(t)-\overline{a(t)}$ and $b(t)-\overline{b(t)}$ are both zero, or $\zeta$ is real. So $\widetilde{H}$ is contained in a real subvariety 
locally. To show that $\widetilde{H}$ is a subvariety at $p$, we need to show that the complexified image does not introduce any points on "the diagonal" that are not in $\widetilde{H}$.

The derivatives of $a$ and $b$ do not both vanish, so without loss of generality $b^{\prime}(0) \neq 0$. Then $b(t)-\overline{b(t)}=0$ implies $t$ is real. Therefore the image of the complexified mapping lies in $\mathbb{C}^{2}$ (on the diagonal), if either $t$ is real or if $\zeta$ is real. A point where $t$ is real is in the image of $(a(t)+\zeta b(t), \zeta)$ for a real $t$ (and therefore in $\widetilde{H}$ ). A point where $\zeta$ is real is in $\Delta$ and therefore in $\widetilde{H}$. Either way the image of $(a(t)+\zeta b(t), \zeta)$ for a real $t$ together with $\Delta$ is a subvariety. Therefore, $\widetilde{H}$ is a subvariety.

We now use this construction to prove Theorem 1.5 .

Proof of Theorem 1.5. Let $X \subset \mathbb{R}^{2}$ be a connected compact real-analytic curve not contained in any proper real-algebraic subvariety of $\mathbb{R}^{2}$. That is, any bihomogeneous polynomial $p(x, y)$ vanishing on $X$, vanishes identically.

Construct $\widetilde{H}$ as in Lemma 5.1. To prove the theorem, we need to show that a polynomial vanishing on $\overline{\widetilde{H}^{*}}$ is the zero polynomial.

Fix two real distinct numbers $\theta_{1}$ and $\theta_{2}$ and two positive real numbers $r_{1}$ and $r_{2}$. Then the set $\overline{\widetilde{H}^{*}} \cap\left\{z \in \mathbb{C}^{3}: z_{1}=r_{1} e^{i \theta_{1}}, z_{2}=r_{2} e^{i \theta_{2}}\right\}$ contains the set

$$
X^{\prime}=\left\{z \in \mathbb{C}^{3}: z_{0}=r_{1} e^{i \theta_{1}} x+r_{2} e^{i \theta_{2}} y \text { where }(x, y) \in X, z_{1}=r_{1} e^{i \theta_{1}}, z_{2}=r_{2} e^{i \theta_{2}}\right\} .
$$

$X^{\prime}$ is a linear image of $X$ under a nonsingular linear mapping. As $X$ is nonalgebraic then $X^{\prime}$ is nonalgebraic, and hence any real polynomial $P$ that vanishes on $\widetilde{H}^{*}$ vanishes on $X^{\prime}$ and hence vanishes on the entire set $\left\{z \in \mathbb{C}^{3}: z_{1}=r_{1} e^{i \theta_{1}}, z_{2}=r_{2} e^{i \theta_{2}}\right\}$. By varying $\theta_{1}$ and $\theta_{2}$ we get that $P$ vanishes on an open subset of $\mathbb{C}^{3}$ and thus vanishes identically.

In the proof of the lemma, note that not all of $\Delta$ was needed, it was only used to cover the points where the image of the map could change depending on what neighbourhood of the complexified variables we took in the local argument above. In fact $\Delta$ is an entire Levi-flat hypersurface. We only need to take the smallest subvariety that includes $\widetilde{H} \backslash \Delta$. Then the subvariety induced by $X$ is the set $\left\{z \in \mathbb{C}^{3}: z_{0}=z_{1} x+z_{2} y\right.$ where $\left.(x, y) \in X\right\}$ together with a subset of $\Delta$.

Corollary 5.2. There exists a singular Levi-flat hypersurface $H \subset \mathbb{P}^{2}$ that is a semialgebraic set, but is not real-algebraic. That is, there exists a real-algebraic Levi-flat subvariety of codimension 1 in $\mathbb{P}^{2}$ that is irreducible as a real-algebraic subvariety, but reducible as a realanalytic subvariety into two components of codimension 1.

Proof. The key is to find an algebraic curve with such properties in $\mathbb{R}^{2}$ and apply Lemma 5.1 . For example, the curve given by

$$
x(x-1)(x-2)(x-3)+y^{2}=0
$$

has two components as a real-analytic subvariety, but is irreducible as a real-algebraic subvariety. Taking only one of those components we construct our $\widetilde{H}$. This is clearly a proper subset of the algebraic set that contains $\widetilde{H}$, which must contain both components. To construct the full algebraic set we simply solve $z_{0}=z_{1} x+z_{2} y, \bar{z}_{0}=\bar{z}_{1} x+\bar{z}_{2} y$, for $x$ and $y$ in terms of rational functions of $z$ and $\bar{z}$, which we plug into the equation $x(x-1)(x-2)(x-3)+y^{2}=0$ and clear denominators. 
The proof that the construction in this note produces a real-analytic subvariety depends on the dimension $n=2$. It is reasonable to conjecture that a similar construction ought to produce a subvariety in higher dimension.

Finally, let us remark why the Levi-foliation does not extend for hypersurfaces constructed above, a fact already clear from the theorem in [12], but let us identify explicitly the points where no extension exists. Without loss of generality and possibly translating $X$, we assume that for all small enough $x \in \mathbb{R}$, there exist at least two distinct $y \in \mathbb{R}$ such that $(x, y) \in X$. Given any real $s_{2}$, then for all sufficiently small real $s_{0}$, there are at least two distinct leaves of the hypersurface $H$ passing through $\left[s_{0}, 1, s_{2}\right]$. These points form a generic totallyreal submanifold, biholomorphic to $\mathbb{R}^{2}$. This set would have to lie in the singular set of any holomorphic foliation tangent to $H$ (that is, extending the Levi-foliation) near $[0,1,0]$, which is impossible, as the singular set of a holomorphic foliation is a complex analytic subvariety.

\section{REFERENCES}

[1] Eric Bedford, Holomorphic continuation of smooth functions over Levi-flat hypersurfaces, Trans. Amer. Math. Soc. 232 (1977), 323-341. MR0481100

[2] Edward Bierstone and Pierre D. Milman, Semianalytic and subanalytic sets, Inst. Hautes Études Sci. Publ. Math. 67 (1988), 5-42. MR0972342

[3] Marco Brunella, Singular Levi-flat hypersurfaces and codimension one foliations, Ann. Sc. Norm. Super. Pisa Cl. Sci. (5) 6 (2007), no. 4, 661-672. MR2394414

[4] _ Some remarks on meromorphic first integrals, Enseign. Math. (2) 58 (2012), no. 3-4, 315-324, DOI 10.4171/LEM/58-3-3. MR3058602

[5] Daniel Burns and Xianghong Gong, Singular Levi-flat real analytic hypersurfaces, Amer. J. Math. 121 (1999), no. 1, 23-53. MR1704996

[6] D. Cerveau and A. Lins Neto, Local Levi-flat hypersurfaces invariants by a codimension one holomorphic foliation, Amer. J. Math. 133 (2011), no. 3, 677-716, DOI 10.1353/ajm.2011.0018. MR2808329

[7] Wei-Liang Chow, On compact complex analytic varieties, Amer. J. Math. 71 (1949), 893-914. MR0033093

[8] Francesco Guaraldo, Patrizia Macrì, and Alessandro Tancredi, Topics on real analytic spaces, Advanced Lectures in Mathematics, Friedr. Vieweg \& Sohn, Braunschweig, 1986. MR1013362

[9] Arturo Fernández-Pérez, On Levi-flat hypersurfaces with generic real singular set, J. Geom. Anal. 23 (2013), no. 4, 2020-2033, DOI 10.1007/s12220-012-9317-1. MR3107688

[10] _ Levi-flat hypersurfaces tangent to projective foliations, J. Geom. Anal. 24 (2014), no. 4, 19591970, DOI 10.1007/s12220-013-9404-y. MR3261727

[11] Arturo Fernández-Pérez, Rogério Mol, and Rudy Rosas, On the dynamics of foliations in $\mathbb{P}^{n}$ tangent to Levi-flat hypersurfaces. preprint arXiv:1403.4802.

[12] Jiří Lebl, Algebraic Levi-flat hypervarieties in complex projective space, J. Geom. Anal. 22 (2012), no. 2, 410-432, DOI 10.1007/s12220-010-9201-9. MR2891732

[13] _ Singular set of a Levi-flat hypersurface is Levi-flat, Math. Ann. 355 (2013), no. 3, 1177-1199, DOI 10.1007/s00208-012-0821-1. MR3020158

[14] _ An interesting example of a compact non-C্-analytic real subvariety of $\mathbb{R}^{3}$. preprint arXiv:1412.4838

[15] Alcides Lins Neto, A note on projective Levi flats and minimal sets of algebraic foliations, Ann. Inst. Fourier (Grenoble) 49 (1999), no. 4, 1369-1385. MR1703092

[16] Rasul Shafikov and Alexandre Sukhov, Germs of singular Levi-flat hypersurfaces and holomorphic foliations. preprint arXiv:1405.4274.

[17] Hassler Whitney, The general type of singularity of a set of $2 n-1$ smooth functions of $n$ variables, Duke Math. J. 10 (1943), 161-172. MR0007784

Department of Mathematics, Oklahoma State University, Stillwater, OK 74078, USA E-mail address: lebl@math.okstate.edu 